\title{
Multiorgan involvement of Behçet's disease in a young woman
}

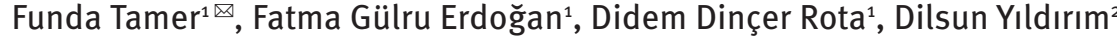

\begin{abstract}
Behçet's disease is a rare disorder of unknown etiology that is classified as a systemic vasculitis. The prevalence of the disease is high in countries in the Far East, Mediterranean Basin, and East Asia. Thus, it is also known as the Silk Road Disease. Behçet's disease is characterized by recurrent oral aphthous ulcers, genital sores, and ocular lesions. However, it can present with severe clinical manifestations as a result of cardiovascular system, central nervous system, and gastrointestinal tract involvement. The disease causes serious complications, morbidity, and mortality, especially in male patients with early age onset. Here we present a rare case of Behçet's disease exhibiting multiple organ involvement in a 26 -year-old Caucasian female.
\end{abstract}

Keywords: Behçet’s disease, management, vasculitis

Received: 19 October 2017 | Returned for modification: 5 January 2018 | Accepted: 10 January 2018

\section{Introduction}

Behçet's disease is a systemic vasculitis that can affect all sizes and types of blood vessels. The disease has a chronic course with exacerbations and remissions. In most cases, Behçet's disease starts with recurrent oral ulcers. However, ocular, neurological, cardiovascular, articular, and gastrointestinal involvement can lead to serious complications and even death. No specific diagnostic tool or disease-specific biomarkers have been identified yet. The diagnosis of Behçet's disease is made by excluding other possible conditions. Cutaneous findings of Behçet's disease include papulopustular and acne-like lesions on the face, limbs, trunk, and buttocks; thrombosis and vasculitis; erythema nodosum lesions in the lower limbs; and cutaneous ulcers in the neck, breast, axillae, inguinal region, and legs that heal with scarring. Therefore, dermatologists play an important role in the diagnosis and management of the disease (1).

\section{Case report}

A 26-year-old Caucasian female presented with a 16-year history of recurrent oral aphthous ulcers. The lesions had been occurring once a month and healed spontaneously within 2 weeks. The patient revealed that she had had painful genital ulcers 2 years earlier. The pathergy phenomenon was positive. Therefore, she was diagnosed with Behçet's disease in 2015. Since then, she had erythema nodosum on the legs, and papulopustular rash on the arms, thighs, and abdominal region. The patient had arthralgia in the left ankle and lower back pain for the previous 10 months. She was diagnosed with sacroiliitis. Furthermore, she had pain, redness, and blurry vision in the left eye 4 months earlier and she was diagnosed with anterior uveitis. The patient had been treated with oral colchicine $1.5 \mathrm{mg} /$ day for the previous 2 years, oral sulfasalazine $1.5 \mathrm{~g}$ /day and indomethacin $75 \mathrm{mg} /$ day for the previous 6 months, and dexamethasone eyedrops $0.1 \mathrm{mg} / 5 \mathrm{ml}$ three times a day for the previous 4 weeks. However, no clinical improvement had been achieved. Physical examination revealed hypopigmented atrophic scars on the perineal region (Fig. 1). The patient was hospitalized for further clinical evaluation. Cranial magnetic resonance angiography showed narrowing of the transverse sinus and sigmoid sinus. The complete blood count, chemistry panel, and serum levels of ferritin, C-reactive protein, and thyroid-stimulating hormone were all within normal limits. However, the patient developed chest pain when breathing. Pulmonary function tests revealed reduced pulmonary diffusion, and chest computed tomography revealed thromboembolic changes on the lateral basal segment of the left lower lobe. Serum D-dimer level was 228 $\mathrm{ng} / \mathrm{ml}$ (normal range: $10-198 \mathrm{ng} / \mathrm{ml}$ ). Serum troponin I, creatine kinase-MB level, electrocardiogram, and echocardiogram were all within normal limits. Therefore, a diagnosis of pulmonary thromboembolism was made and the patient was started on oral prednisolone $60 \mathrm{mg} /$ day and enoxaparin sodium $0.6 \mathrm{mg} /$ day. Significant clinical improvement was achieved and the steroid dose was gradually reduced. After prednisolone and enoxaparin therapy, she was started on oral azathioprine $100 \mathrm{mg} /$ day and colchicine $1.5 \mathrm{mg} /$ day. Close follow-up was recommended for the patient.

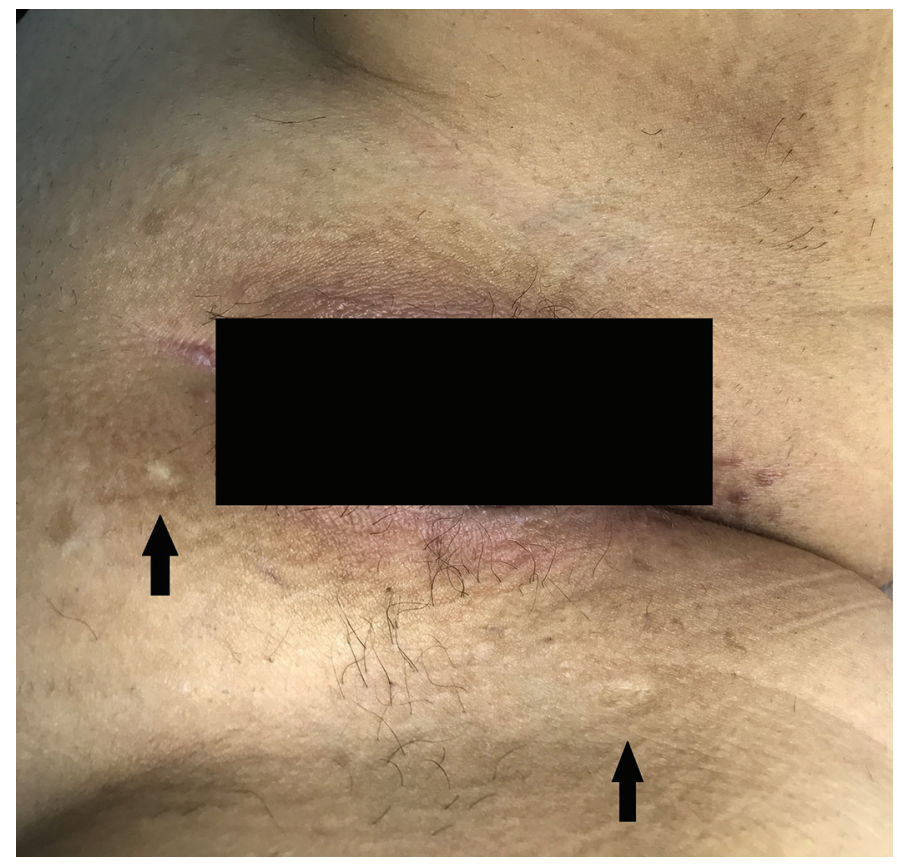

Figure 1 | Hypopigmented atrophic scars on the perineum. 


\section{Discussion}

Behçet's disease is a systemic vasculitic disorder that is characterized by recurrent oral and genital ulcers, uveitis, skin lesions such as papulopustular lesions, erythema nodosum, and ocular, vascular, gastrointestinal, and nervous system complications (2, 3). The condition was recognized by Hippocrates in the fifth century BC and Adamantiades in 1931. However, the classical triad of oral and genital ulcers with intraocular inflammation was first reported by Hulusi Behçet (4). Therefore, synonyms such as triple symptom complex, mucocutaneous-ocular syndrome, Adamantiades-Behçet's syndrome, and Adamantiades-Behçet's disease have been used for this condition (5).

The prevalence of the disease in Turkey is 80 to 370 cases per 100,000 (6). Behçet's disease usually occurs around age 30. It affects men and women equally (7). Ocular disease, papulopustular lesions, venous thrombosis, heart disease, and positive pathergy are more common in male patients, whereas genital ulcers, erythema nodosum, and joint involvement are associated with females (8). It is difficult to predict its course, prognosis, and response to treatment (1). However, gender influences the prognosis. The course of the disease is usually more severe in men than in women as a result of frequent vital organ involvement in male patients (8). Behçet's disease tends to remit within 5 to 10 years. It can also result in numerous comorbidities such as blindness, physical disability, cognitive impairment, and increased mortality (7). Successful management of Behçet's disease requires a multidisciplinary approach (6). The treatment plan should be made based on the disease severity and organ involvement. Therapeutic options include tumor necrosis factor- $a$ inhibitors, interferon- $a$, apremilast, and traditional treatments such as corticosteroids, non-steroidal anti-inflammatory drugs, colchicine, azathioprine, mycophenolate mofetil, cyclosporine, tacrolimus, cyclophosphamide, thalidomide, methotrexate, and dapsone (9). However, poor adherence to therapy has been reported in patients with Behçet's disease. Male sex, lower income, unemployment, unawareness of the disease complications, and therapy with non-steroidal anti-inflammatory drugs have been reported to be associated with non-adherence to treatment (10).

The patient we presented is a good example of an aggressive course of Behçet's disease with multiple organ involvement. She had recurrent oral ulcerations and ocular, joint, pulmonary, and neurological manifestations. Despite systemic colchicine and sulfasalazine therapy, the disease progressed rapidly. We report this case because of the rarity of severe complications of Behçet's disease in young women.

\section{References}

1. Zeidan MJ, Saadoun D, Garrido M, Klatzmann D, Six A, Cacoub P. Behçet's disease physiopathology: a contemporary review. Auto Immun Highlights. 2016; $7: 4$.

2. Upala S, Yong WC, Sanguankeo A. Increased arterial stiffness in Behçet's disease: a systematic review and meta-analysis. Korean Circ J. 2017;47:477-82.

3. Esatoglu SN, Kutlubay Z, Ucar D, Hatemi I, Uygunoglu U, Siva A, et al. Behçet's syndrome: providing integrated care. I Multidiscip Healthc. 2017;10:309-19.

4. Kontogiannis V, Powell RJ. Behçet's disease. Postgrad Med J. 2000;76:629-37.

5. Cho SB, Cho S, Bang D. New insights in the clinical understanding of Behçet's disease. Yonsei Med J. 2012;53:35-42.
6. Nair JR, Moots RJ. Behcet's disease. Clin Med (Lond). 2017;17:71-7.

7. Seyahi E. Behçet's disease: how to diagnose and treat vascular involvement. Best Pract Res Clin Rheumatol. 2016;30:279-95.

8. Scherrer MAR, Rocha VB, Garcia LC. Behçet's disease: review with emphasis on dermatological aspects. An Bras Dermatol. 2017;92:452-64.

9. Saleh Z, Arayssi T. Update on the therapy of Behçet disease. Ther Adv Chronic Dis. 2014;5:112-34.

10. Khabbazi A, Karkon Shayan F, Ghojazadeh M, Kavandi H, Hajialiloo M, Esalat Manesh K, et al. Adherence to treatment in patients with Behcet's disease. Int J Rheum Dis. 2017 [Epub ahead of print]. 